\title{
Spectroscopic signatures of proton transfer dynamics in the water dimer cation
}

\author{
Eugene Kamarchik ${ }^{a, b}$, Oleg Kostko ${ }^{c}$, Joel M. \\ Bowman $^{b}$, Musahid Ahmed ${ }^{c}$, and Anna I. Krylov ${ }^{a}$ \\ ${ }^{a}$ Department of Chemistry, University of Southern California, \\ Los Angeles, CA 90089-0482, USA \\ ${ }^{b}$ C.L. Emerson Center for Scientific Computation, Dept. of Chemistry, \\ Emory University, Atlanta, GA 30322 \\ ${ }^{c}$ Chemical Sciences Division, Lawrence Berkeley National Laboratory, Berkeley, CA 94720, USA
}

(Dated: March 11, 2010)

Using full dimensional EOM-IP-CCSD/aug-cc-pVTZ potential energy surfaces, the photoelectron spectrum, vibrational structure, and ionization dynamics of the water dimer radical cation, $\left(\mathrm{H}_{2} \mathrm{O}\right)_{2}^{+}$, were computed. We also report an experimental photoelectron spectrum which is derived from photoionization efficiency measurements and compares favorably with the theoretical spectrum. The vibrational structure is also compared with the recent experimental work of Gardenier et. al. [J. Phys. Chem. A 113, 4772 (2009)] and the recent theoretical calculations by Cheng et. al. [J. Phys. Chem. A 11313779 (2009)]. A reduced dimensionality nuclear Hamiltonian was used to compute the ionization dynamics for both the ground state and first excited state of the cation. The dynamics show markedly different behavior and spectroscopic signatures depending on which state of the cation is accessed by the ionization. Ionization to the ground-state cation surface induces a hydrogen transfer which is complete within 50 femtoseconds, whereas ionization to the first excited state results in a much slower process.

\section{INTRODUCTION}

Structure and dynamics in hydrogen-bonded systems have been of great interest to both theoretical and experimental chemists owing to the important role these systems play in biological, chemical, and atmospheric science[1-5]. For example, hydrogen bonding is re- 
sponsible for the double-helix structure of the DNA and for many unique properties of the most important solvent, water. Hydrogen bonds can facilitate hydrogen or proton transfer between molecules (or within the same molecule), a process commonly occurring in biochemistry, enzyme catalysis, and materials science. Proton transfer is often coupled to electronic dynamics, and can be induced by electronic excitation or ionization. For example, ionization of some hydrogen-bonded nucleobase pairs leads to barrierless proton transfer, which is believed to play a role in the hole migration through the DNA[6-14]. Reduced barriers along proton transfer coordinate have also been reported in electronically excited nucleobase pairs $[15,16]$.

Ionization of liquid water, which has been investigated extensively since the 1960s, also involves proton transfer, however, the entire process is still not clearly understood. Experimentally, it is observed that ionizing radiation strips an electron from water, resulting in a thermalized electron, a hydroxyl radical $(\mathrm{OH})$, and a hydronium ion $\left(\mathrm{H}_{3} \mathrm{O}^{+}\right)$. It is believed that this results from the formation of a nascent water cation $\left(\mathrm{H}_{2} \mathrm{O}^{+}\right)$, which subsequently reacts by transferring a proton along one of its hydrogen bonds to a neighboring water monomer. The water dimer cation is a minimal model system (readily amendable to experimental and theoretical studies) that exhibits similar photoinduced dynamics. In the neutral state, the water dimer exists in the classic donor-acceptor configuration with one of the water monomers oriented so that it points a hydrogen at the second water. Ionization of the dimer induces a proton transfer reaction[1]. Representing the vertically ionized water dimer as $\left[\left(\mathrm{H}_{2} \mathrm{O}\right)_{2}\right]^{+}$and the proton-transferred product as a $\mathrm{H}_{3} \mathrm{O}^{+} \cdot \mathrm{OH}$ pair, the reaction can be written as:

$$
\left(\mathrm{H}_{2} \mathrm{O}\right)_{2}+\mathrm{h} \nu \rightarrow\left[\left(\mathrm{H}_{2} \mathrm{O}\right)_{2}\right]^{+}+\mathrm{e}^{-} \rightarrow\left(\mathrm{H}_{3} \mathrm{O}\right)^{+} \ldots \mathrm{OH} \rightarrow\left(\mathrm{H}_{3} \mathrm{O}\right)^{+}+\mathrm{OH}
$$

The $\mathrm{H}_{3} \mathrm{O}^{+} \cdot \mathrm{OH}$ complex is a bound complex, and may or may not dissociate depending on the energy of the impinging radiation. In bulk water, the proton transfer is believed to be complete within 100 femtoseconds and has been followed by transient electronic spectroscopy[17], although it has been difficult to establish definitive evidence for this transformation.

As a prototypical H-bonded system, the water dimer is of great fundamental interest [18, 19]. While the dynamics and vibrational spectroscopy (including hydrogen tunneling) of the neutral water dimer has been extensively studied [20-22], previous dimer cation work has 
primarily focused on the controversy relating to the energetic minimum of the cation [2327], although the dynamics on the surface have also been attempted[28]. Only recently has theoretical work begun to address other features of the cation, such as the nature of the initially formed hole, $\mathrm{H}_{2} \mathrm{O}_{(\mathrm{aq})}^{+}$. The hole delocalization depends strongly on dimer geometry, revealing strong couplings between the states of the hydrogen-bond donor and acceptor $[29,30]$. The structures, vibrational frequencies, and energetics of the ground-state of the water dimer cation have also been newly characterized computationally[31]. At the same time, recent experimental work has addressed the vibrational structure of the bound complex $\mathrm{H}_{3} \mathrm{O}^{+} \cdot \mathrm{OH}$ with the assignment of several important fundamental frequencies[32].

The photoelectron spectrum of the water dimer has been reported by Tomoda et al[33]. Assuming that the dimers are the dominant species in the beam, the reported spectrum was obtained as a difference between the photoelectron spectrum of all the species in the beam and the spectrum of the water monomer. The spectrum features two bands with maxima at 12.1 and $13.2 \mathrm{eV}$, respectively, the first band being visibly broader than the second. The onset of the first band was $11.1 \mathrm{eV}$. An earlier photoionization study of $\mathrm{Ng}$ et al.[34] reported an adiabatic IE of $11.21 \mathrm{eV}$. In view of large geometric relaxation and unfavorable FranckCondon factors, the authors regarded this value as an upper bound of the AIE. This study also reported the onset for $\mathrm{H}_{3} \mathrm{O}^{+}$appearance at $11.7 \mathrm{eV}$, and gave a lower bound for the dimer dissociation (to $\mathrm{H}_{2} \mathrm{O}^{+}+\mathrm{H}_{2} \mathrm{O}$ ) as $1.58 \mathrm{eV}$. A more recent study using a charge-exchange reaction[35] reported lower values for the AIE, i.e., 10.8-10.9 eV. Valence ionization of water clusters and size dependence of the IEs has been recently investigated[36].

The focus of this paper is on ionization-induced dynamics in water dimer and on characterizing spectroscopic signatures of proton transfer. We compare proton-transfer dynamics in the two different electronic states of the cation and investigate how the evolution of the ionized dimer depends on the character of the ionized state. Our calculations employ fulldimensional potential energy surfaces (PES) computed by the equation-of-motion coupledcluster (EOM-CC) method for ionization potentials (EOM-IP-CC)[37-41]. We discuss the photoionization of the dimer from two different perspectives. First, we report calculations of vibrational wave functions of the cation and present photoelectron spectrum of $\left(\mathrm{H}_{2} \mathrm{O}\right)_{2}$. Photoelectron spectra contain the information about energies and the character of the electronic states of the ionized system as well as ionization-induced dynamics. For example, long Franck-Condon progressions reveal significant geometry changes, from which dynami- 
cal information can be deduced. By comparing the spectra of the monomer and the dimer, one can quantify the effects of clustering on electronic states, such as shifts in ionization energies (see, for example, Refs. [13, 42, 43]). We compare the computed photoelectron spectrum of the dimer with the pseudo-photoelectron spectrum derived from photo efficiency measurements. The spectrum reveals important differences in hole localization and the vibrational structure of the complex in the lowest and the first excited electronic states, which are critical to understanding the proton transfer dynamics. The onset and overall shape of the first band of the computed spectrum agrees well with the PIE derived one. The computed vibrational frequencies for the ground state of the cation also agree well with the recent experimental results[32].

Second, we present results of wave packet propagation on each of the two potential surfaces of the water dimer cation. These calculations provide an insight into the timescale and dynamics of the ensuing proton transfer process. In agreement with different FranckCondon envelops, we observe different time scales for proton transfer in the two ionized states. Moreover, we investigate the evolution of the electronic spectrum during the hydrogen transfer process in the two electronic states. Different patterns in the electronic spectral evolution can be exploited in pump-probe experiments to distinguish between electronic states of the dimer cation.

Despite its modest size, the water dimer cation presents several challenges for computational methods. From the electronic structure point of view, its open-shell doublet wave functions are difficult to describe by standard single-reference approaches due to spincontamination and symmetry breaking persisting even when highly correlated methods are employed[40]. Density functional theory suffers from self interaction error, which results in overestimation of the delocalization of the hole and underestimation of the barriers for proton transfer. Modeling nuclear dynamics in this system needs to properly account for quantum effects (i.e, zero-point motion) and large anharmonicities, which are important owing to the presence of light atoms (hydrogens) and weak inter-fragment interactions.

We employ EOM-IP-CC[37-41], which is the method of choice for ionized systems[44]. It is capable of describing multiple interacting electronic states in a balanced and accurate fashion, and is free of spin-contamination and symmetry breaking. Moreover, EOM-IPCCSD allows one to compute transition properties required for spectroscopy modeling, e.g., transition dipole moments and Dyson orbitals[45, 46]. Using EOM-IP-CCSD, we compute 
a full-dimensional semi-global PES using an invariant polynomial representation [47] as described below. These surfaces are used for vibrational self-consistent field/vibrational configuration interaction (VSCF/VCI) calculations and reduced-dimensionality wave packet propagation. The wave packet calculations employ an approximate kinetic energy operator, which we also derive below.

The structure of the paper is as follows. In the next section we describe the calculation of the required ground and excited state potential energy surfaces for the water dimer cation. This section also includes the derivation of and our motivation for a particular kinetic energy operator in internal coordinates. Section III presents the details of the experimental photoionization efficiency measurements to which we will compare our theoretical results. Section IV tabulates both experimental and theoretical results, presenting the potential energy surfaces, the vibrational structure, the photoelectron spectrum, the wavepacket dynamics, and the spectral evolution. Finally, we conclude with a discussion of the differences between dynamics on different surfaces as well as presenting some insight into future comparisons between theory and experiment.

\section{THEORETICAL METHODS AND COMPUTATIONAL DETAILS}

The full-dimensional PESs of the ground and first excited states of the water dimer cation were fit to electronic energies computed with EOM-IP-CC with single and double substitutions (EOM-IP-CCSD)[37-41] with the aug-cc-pVTZ basis set [48] using the Q-CHEM suite of quantum chemistry programs[49]. The core electrons are frozen in all calculations. Previous EOM-IP calculations employed the $6-311++\mathrm{G}^{* *}$ basis set[30], and our results demonstrate that using a larger basis set effects the IEs by as much as $0.3 \mathrm{eV}$, resulting in better agreement with the experimental values.

The PESs were constructed using 13,169 and 9,137 single point energies for the ground state and first excited state, respectively. The geometries were chosen such that they are in the configuration space that is sampled by the vertical ionization and subsequent proton transfer. This choice was based on two criteria, namely, to accurately describe the potential in the region near the global minimum of the cation in order to permit accurate vibrational calculations and in the four-dimensional subspace spanned by the coordinates we are interested in for the dynamics. The fitting was done using a $6^{\text {th }}$ degree polynomial constructed 
from permutationally invariant polynomials in Morse variables. The Morse variables are defined as $y(i, j)=e^{\frac{-r(i, j)}{\lambda}}$, where $r(i, j)$ is the internuclear distance between atoms $i$ and $j$. The value of $\lambda$ was chosen to be $2.0 \AA$, however, the fit is relatively insensitive to this value. The overall total root-mean-square error for the ground state and first excited state surfaces were $264 \mathrm{~cm}^{-1}$ and $512 \mathrm{~cm}^{-1}$ respectively. The PES of the neutral dimer was constructed from 10,240 single point energies from the same set of calculations and had a RMS error of $80 \mathrm{~cm}^{-1}$. In the region spanning the four-dimensional subspace for the wavepacket dynamics, the neutral, ground state cation, and first excited state cation surfaces had RMS errors of $182 \mathrm{~cm}^{-1}, 311 \mathrm{~cm}^{-1}$, and $393 \mathrm{~cm}^{-1}$ respectively. Since this region spans a considerable range of energies and some contribution to the RMS error comes from enforcing the correct behavior as nuclei become close, we expect that these errors will not significantly impact the overall accuracy of the calculation. The PESs employ the ezPES[50] interface and are available for download from the iOpenShell website.

Using the PES VSCF/CVI calculations were performed in order to extract the ground state vibrational wave function as well as excited state energies of the bound complex. Both of these methods are well documented[51, 52]. The vibrational calculations employed the $n$-mode representation of the potential at the 3 -mode level and each mode employed a primitive sinc-discrete variable representation (sinc-DVR) [53-55] basis of 20 points, to diagonalize the Watson Hamiltonian [56] yielding the vibrational states.

In the second set of calculations, aimed at wavepacket dynamics, we restrict our attention to a four-dimensional subset of the twelve internal coordinates. In order to clarify subsequent presentation of the kinetic energy operator we adopt the following notation based on the neutral water dimer geometry: $\mathrm{H}_{\mathrm{a}}$ refers to either hydrogen in the acceptor monomer and $\mathrm{O}_{\mathrm{a}}$ refers to the oxygen in the acceptor, the three remaining atoms are labelled $\mathrm{H}_{\mathrm{s}}$ for the shared proton, $\mathrm{O}_{\mathrm{d}}$ for the donor oxygen, and $\mathrm{H}_{\mathrm{f}}$ for the free hydrogen on the donor. Figure 1 demonstrates this labeling. In this case, we evaluated cuts through the PES allowing the distance between the shared proton and the oxygen in the donor water, $r_{\mathrm{O}_{\mathrm{d}} \mathrm{H}_{\mathrm{s}}}$, the oxygenoxygen distance, $r_{\mathrm{O}_{\mathrm{d}} \mathrm{O}_{\mathrm{a}}}$, the bond angle between the two hydrogens in the hydrogen-bond acceptor, $\theta_{\mathrm{H}_{\mathrm{a}} \mathrm{O}_{\mathrm{a}} \mathrm{H}_{\mathrm{a}}}$, and the angle corresponding to the rotation of the free hydrogen in the $\mathrm{OH}$ unit about the $\mathrm{O}_{\mathrm{d}} \mathrm{H}_{\mathrm{s}}$ bond, $\tau_{\mathrm{H}_{\mathrm{f}} \mathrm{OdH}_{\mathrm{s}} \mathrm{O}_{a}}$, to vary. All remaining internal coordinates were held at their equilibrium values for the neutral water dimer $\left(r_{\mathrm{O}_{\mathrm{d}} \mathrm{H}_{\mathrm{f}}}=0.958 \AA, r_{\mathrm{O}_{\mathrm{a}} \mathrm{H}_{\mathrm{a}}}=\right.$ $\left.0.960 \AA, \theta_{\mathrm{H}_{\mathrm{f}} \mathrm{O}_{\mathrm{d}} \mathrm{H}_{\mathrm{s}}}=104.6^{\circ}, \theta_{\mathrm{O}_{\mathrm{a}} \mathrm{O}_{\mathrm{d}} \mathrm{H}_{\mathrm{s}}}=3.3^{\circ}, \theta_{\mathrm{H}_{\mathrm{a}} \mathrm{O}_{\mathrm{a}} \mathrm{O}_{\mathrm{d}}}=110.5^{\circ}, \tau_{\mathrm{H}_{\mathrm{a}} \mathrm{O}_{\mathrm{a}} \mathrm{H}_{\mathrm{s}} \mathrm{O}_{\mathrm{d}}}=58.0^{\circ}\right)$. While the 
VSCF and VCI calculations employed the Watson Hamiltonian in rectilinear coordinates, for dynamics calculations we employed the above curvilinear valence coordinates.

While the use of curvilinear coordinates allows for a more accurate description of large scale displacements, it significantly increases the complexity of the kinetic energy operator. For this reason, we constructed an approximate kinetic energy operator $\hat{T}$ by constraining the atomic motions to representative planes. Although for a six atom system it is both algebraically and computationally feasible to derive the exact constrained kinetic energy operator, we adopt the approximate operator because we believe that it retains the most pertinent features while having much lower complexity and significantly greater ease of implementation.

To construct the kinetic energy operator, we first consider the two oxygen atoms and the bridging hydrogen atom. Within this subsystem, the angle is fixed and only the two bond lengths, $r_{\mathrm{O}_{\mathrm{d}} \mathrm{H}_{\mathrm{s}}}$ and $r_{\mathrm{O}_{\mathrm{d}} \mathrm{O}_{\mathrm{a}}}$, are allowed to vary. This leads to the operator (in atomic units)

$$
\hat{T}_{\text {bond }}=-\frac{1}{\mu_{\mathrm{OO}}} \frac{\partial^{2}}{\partial r_{\mathrm{O}_{\mathrm{d}} \mathrm{O}_{\mathrm{a}}}^{2}}-\frac{1}{\mu_{\mathrm{OH}}} \frac{\partial^{2}}{\partial r_{\mathrm{O}_{\mathrm{d}} \mathrm{H}_{\mathrm{s}}}^{2}}-\frac{\cos \left(\theta_{\mathrm{O}_{\mathrm{a}} \mathrm{O}_{\mathrm{d}} \mathrm{H}_{\mathrm{s}}}\right)}{m_{\mathrm{O}}} \frac{\partial^{2}}{\partial r_{\mathrm{O}_{\mathrm{d}} \mathrm{O}_{\mathrm{a}}} \partial r_{\mathrm{O}_{\mathrm{d}} \mathrm{H}_{\mathrm{s}}}},
$$

where $\mu_{\mathrm{OO}}$ is the reduced mass corresponding to two oxygen atoms and $\mu_{\mathrm{OH}}$ is the reduced mass corresponding to an oxygen and a hydrogen atom[32, 57]. In order to in-

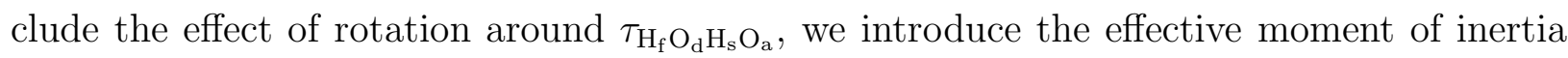
$I_{\tau}=m_{\mathrm{H}} r_{\mathrm{O}_{\mathrm{d}} \mathrm{H}_{\mathrm{f}}}^{2} \sin ^{2}\left(\theta_{\mathrm{H}_{\mathrm{S}} \mathrm{OH}_{\mathrm{f}}}-\theta_{\mathrm{O}_{\mathrm{a}} \mathrm{O}_{\mathrm{d}} \mathrm{H}_{\mathrm{s}}}\right)$, which describes the dihedral rotation of the hydrogen on the hydroxyl fragment. This simple first-order approximation arises from decoupling the torsional motion from the stretching motions involving the donor oxygen atom. The choice of angle $\theta_{\mathrm{H}_{\mathrm{s}} \mathrm{O}_{\mathrm{d}} \mathrm{H}_{\mathrm{f}}}-\theta_{\mathrm{O}_{\mathrm{a}} \mathrm{O}_{\mathrm{d}} \mathrm{H}_{\mathrm{s}}}$ is due to the rotation of this hydrogen about the $\mathrm{H}_{\mathrm{s}} \mathrm{O}_{\mathrm{d}}$ axis rather than about the $\mathrm{O}_{a} \mathrm{O}_{d}$ axis. We belive this rotation does a slightly better job of preserving the acceptor geometry, although the difference between the two rotations is small because $\theta_{\mathrm{O}_{\mathrm{a}} \mathrm{O}_{\mathrm{d}} \mathrm{H}_{\mathrm{s}}}$ is almost zero. Based on this reasoning the corresponding kinetic energy operator is given by

$$
\hat{T}_{\text {dihedral }}=-\frac{1}{2 I_{\tau}} \frac{\partial^{2}}{\partial \tau_{\mathrm{H}_{\mathrm{f}} \mathrm{O}_{\mathrm{d}} \mathrm{H}_{\mathrm{s}} \mathrm{O}_{\mathrm{a}}}}
$$

for the dihedral motion. Finally, to describe the angular kinetic energy corresponding to the bond angle, $\theta_{\mathrm{HaO}_{\mathrm{a}} \mathrm{H}_{\mathrm{a}}}$, we introduce the operator

$$
\begin{aligned}
& \hat{T}_{\text {angle }}=\left(-\frac{2}{\mu_{\mathrm{OH}} r_{\mathrm{O}_{\mathrm{a}}}^{2}}+2 \frac{\cos \left(\theta_{\mathrm{H}_{\mathrm{a}} \mathrm{O}_{\mathrm{a}} \mathrm{H}_{\mathrm{a}}}\right)}{m_{\mathrm{O}} r_{\mathrm{O}_{\mathrm{a}} \mathrm{H}_{\mathrm{a}}}}\right)\left(\frac{\partial^{2}}{\partial \theta_{\mathrm{H}_{\mathrm{a}} \mathrm{O}_{\mathrm{a}}}^{2}}+\cot \left(\theta_{\mathrm{H}_{\mathrm{a}} \mathrm{O}_{\mathrm{a}} \mathrm{H}_{\mathrm{a}}}\right) \frac{\partial}{\partial \theta_{\mathrm{H}_{\mathrm{a}} \mathrm{O}_{\mathrm{a}} \mathrm{H}_{\mathrm{a}}}}\right) \\
& -\frac{2 \sin \left(\theta_{\mathrm{H}_{\mathrm{a}} \mathrm{O}_{\mathrm{a}} \mathrm{H}_{\mathrm{a}}}\right)}{m_{\mathrm{O}_{\mathrm{O}_{\mathrm{a}} \mathrm{H}_{\mathrm{a}}}^{2}}} \frac{\partial}{\partial \theta_{\mathrm{H}_{\mathrm{a}} \mathrm{O}_{\mathrm{a}}}}+\sin \left(\theta_{\mathrm{H}_{\mathrm{a}} \mathrm{O}_{\mathrm{a}} \mathrm{O}_{\mathrm{d}}}\right) \cos \left(\tau_{\mathrm{O}_{\mathrm{d}} \mathrm{O}_{\mathrm{a}} \mathrm{H}_{\mathrm{a}} \mathrm{H}_{\mathrm{a}}}\right) \frac{1}{m_{O_{\mathrm{O}} r_{\mathrm{a}}}^{2}} \frac{\partial^{2}}{\partial r_{\mathrm{O}_{\mathrm{d}} \mathrm{O}_{\mathrm{a}}} \partial \theta_{\mathrm{H}_{\mathrm{a}} \mathrm{O}_{\mathrm{a}}}},
\end{aligned}
$$


which arises from the elements of the G-matrix[57]. Combining these three kinetic energy operators with the potential, the Hamiltonian is given by

$$
\hat{H}=\hat{T}_{\text {bond }}+\hat{T}_{\text {dihedral }}+\hat{T}_{\text {angle }}+V\left(r_{\mathrm{O}_{\mathrm{d}} \mathrm{H}_{\mathrm{s}}}, r_{\mathrm{O}_{\mathrm{d}} \mathrm{O}_{\mathrm{a}}}, \tau_{\mathrm{H}_{\mathrm{f}} \mathrm{O}_{\mathrm{d}} \mathrm{H}_{\mathrm{s}} \mathrm{O}_{\mathrm{a}}}, \theta_{\mathrm{H}_{\mathrm{a}} \mathrm{O}_{\mathrm{a}} \mathrm{H}_{\mathrm{a}}}\right) .
$$

The Hamiltonian was evaluated on a direct-product grid using a 20 point sinc-discrete variable representation (sinc-DVR) in each degree of freedom. Expressions for first- and second-derivatives in this basis are readily available in the literature and so are not reproduced here[53-55]. Three-dimensional results obtained using the two bond angles and the dihedral rotation with a 24 point sinc-DVR grid were not appreciably different from a similar calculation with the 20 point grid, so we expect that the basis, although small, is adequate for our desired level of accuracy. In order to reduce the size of the resulting direct-product grid, an energy cutoff $V_{t h r}$ was established, and all grid points having a potential energy above this threshold were removed. For the neutral water dimer, ground state water dimer cation, and first excited state water dimer cation this matrix was evaluated and diagonalized. Since for the neutral water dimer, the only state of interest was the ground vibrational state, this could be accomplished directly by a Lanczos diagonalization routine. However, for the cation a complete set of states in which to expand the ground vibrational state of the neutral was desired. In order to achieve this, a subspace diagonalization and recoupling algorithm

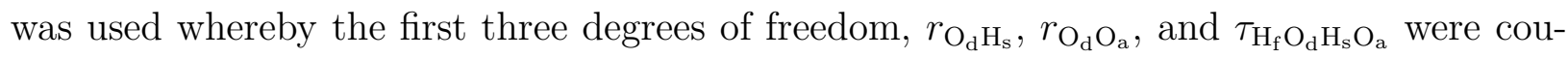
pled. The 500 eigenvectors with the largest overlap with the neutral ground state were then chosen and coupled with the remaining fourth degree of freedom, $\theta_{\mathrm{H}_{\mathrm{a}} \mathrm{O}_{\mathrm{a}} \mathrm{H}_{\mathrm{a}}}$. Quantum dynamics were then performed by expanding the ground state vibrational wave function of the neutral dimer in the eigenbasis of either the ground or first excited state of the cation. This choice of basis is particularly convenient because it diagonalizes the relevant Hamiltonian, allowing the propagator $e^{-\imath \hat{H} t}$ to be calculated efficiently and analytically.

Electronic spectra were computed using EOM-IP-CCSD/aug-cc-pVTZ transition dipole moments at the centroid of the wave packets and are convoluted with the pulse shape, as described in the next section, instead of doing the multidimensional integration. These approximate spectra nevertheless provide a reasonable theoretical estimate which can be compared with experiment.

All relevant geometries and energies as well as computed spectra visualized below are given in the EPAPS supplementary materials[58]. The PES of the two states of the cation 
are available for download from the iOpenShell website[50].

\section{EXPERIMENTAL DETAILS}

The experiments were performed on a molecular beam apparatus coupled to a 3 meter VUV monochromator on the Chemical Dynamics Beamline at the Advanced Light Source (ALS). The clusters were ionized by tunable synchrotron radiation in the $7.4 \mathrm{eV}$ region, and the ions detected by a time-of-flight mass spectrometer. For each mass, the yield of the ions was measured as a function of photon energy, which produced a photoionization efficiency (PIE) spectra. The typical step size for the PIE scans was $50 \mathrm{meV}$, with a dwell time of $10 \mathrm{~s}$ at a repetition rate of $10 \mathrm{kHz}$. The differentiation of the PIE curves, following the method used by Berkowitz in interpreting photoionization of methanol[59], produces a spectrum similar to a photoelectron spectrum from which information about vibrational progressions and other electronic states can be extracted, with caveats relating to the presence of fragmentation channels. The differentiation was performed numerically after taking a five points nearest-neighbor average to reduce the effects of noise in the PIE. The accuracy of reported onset energies in the PIE spectra is $0.05 \mathrm{eV}$.

\section{RESULTS AND DISCUSSION}

Electronic states of the dimer cation can be conveniently described in terms of the dimer molecular orbitals - linear combination of fragment molecular orbitals (DMO-LCFMO) approach $[29,45]$. In this framework we adopt a composite notation of the form (hydrogenbond donor orbital)/(hydrogen-bond acceptor orbital) to describe the dimer molecular orbitals. The three orbitals of the water monomer that will play a role in the spectroscopy are, in order of decreasing energy, the $b_{1}$ (out-of-plane lone pair), $a_{1}$ (in-plane lone pair), and $b_{2}$ (the $\sigma_{\mathrm{OH}}$ bonding orbital). The respective vertical IEs (VIEs) are 11.72 and 13.16 eV (EOM-IP-CCSD/aug-cc-pVTZ) are red-shifted with respect to the monomer's values by

0.91 and $1.77 \mathrm{eV}$, respectively. The previously reported experimental VIEs are 12.1 and $13.2 \mathrm{eV}[33]$, however, since they were derived from a low-resolution difference spectrum, the comparison is not straightforward. The adiabatic IEs are 11.1 and $12.5 \mathrm{eV}$. The lowest AIE 
agrees well with 11.1-11.2 eV values derived from the photoionization and photoelectron measurements[33, 34], and is within $0.3 \mathrm{eV}$ within the lower value of 10.8-10.9 from Refs. $[31,35]$. As shown below, our results are in an excellent agreement with the PIE derived IEs.

\section{A. Potential Energy Surfaces of $\left(\mathrm{H}_{2} \mathrm{O}\right)_{2}^{+}$}

The ground state of the water dimer cation is derived from the ionization at $11.72 \mathrm{eV}$ of an electron from the $b_{1} / 0$ orbital, which corresponds primarily to removing an electron from the lone pair of the hydrogen bond donor. This results in an immediate $\left(\mathrm{H}_{2} \mathrm{O}\right) \cdot\left(\mathrm{H}_{2} \mathrm{O}^{+}\right)$ pair where the hole is localized on one of the water monomers. The localization of this hole creates a strong driving force for the transfer of the shared proton from one monomer to the other yielding the final $(\mathrm{OH}) \cdot\left(\mathrm{H}_{3} \mathrm{O}\right)^{+}$pair.

The equilibrium structure of the ground state cation is significantly different from the equilibrium structure of the neutral water dimer. The three internal coordinates displaying the largest changes are the distance of the shared proton from the $\mathrm{OH}$ oxygen, $r_{\mathrm{OHs}}$, which increases from $0.96 \AA$ to $1.46 \AA$, the oxygen-oxygen distance, $r_{\mathrm{OO}}$, which decreases from $2.85 \AA$ to $2.51 \AA$, and the dihedral angle corresponding to the out-of-plane rotation of the hydrogen in the $\mathrm{OH}$ unit, $\tau_{\mathrm{HOHO}}$, which changes from $180.0^{\circ}$ to $154.1^{\circ}$. Less significant changes are the decrease of the $\theta_{\mathrm{H}_{\mathrm{a}} \mathrm{O}_{\mathrm{a}} \mathrm{H}_{\mathrm{a}}}$ angle of the $\mathrm{H}_{3} \mathrm{O}^{+}$unit and the decrease of the outer $\mathrm{OH}$ bond lengths in the $\mathrm{H}_{3} \mathrm{O}^{+}$unit. The vertical ionization places the $\left(\mathrm{H}_{2} \mathrm{O}\right)_{2}^{+}$approximately $7300 \mathrm{~cm}^{-1}$ above the global minimum on the ground state cation surface. Energetically, the relaxation to the equilibrium structure involves primarily only two coordinates, $r_{\mathrm{OHs}}$ and $r_{\mathrm{OO}}$. Despite the large change in the dihedral angle, the PES is relatively flat along this coordinate. Maintaining this coordinate at the equilibrium geometry of the neutral dimer results in a change of less than $50 \mathrm{~cm}^{-1}$ in potential energy, thus, the energetic contribution of the dihedral angle to the total relaxation is almost negligible.

The coupling between modes introduced by the ionization was investigated by calculating several two- and three-dimensional slices of the ground-state cation surface. Twodimensional slices involving the proton transfer coordinate, $r_{\mathrm{O}_{\mathrm{d}} \mathrm{H}_{\mathrm{s}}}$, and all other possible coordinates reveal a strong coupling between $r_{\mathrm{O}_{\mathrm{d}} \mathrm{H}_{\mathrm{s}}}$ and $r_{\mathrm{O}_{\mathrm{d}} \mathrm{O}_{\mathrm{a}}}$ which was expected from the large displacements. The remaining couplings between $r_{\mathrm{O}_{\mathrm{d}} \mathrm{H}_{\mathrm{s}}}$ and other coordinates are rela- 
tively weak. Likewise, three-dimensional slices including $r_{\mathrm{O}_{\mathrm{d}} \mathrm{H}_{\mathrm{s}}}, r_{\mathrm{O}_{\mathrm{d}} \mathrm{O}_{\mathrm{a}}}$ and a third coordinate did not reveal any strong third-order couplings. The strong coupling between the $r_{\mathrm{O}_{\mathrm{d}} \mathrm{H}_{\mathrm{s}}}$ and $r_{\mathrm{O}_{\mathrm{d}} \mathrm{O}_{\mathrm{a}}}$ coordinates is illustrated in Figure 2. Whereas in the neutral water dimer these two motions are almost completely decoupled in the cation these two coordinates become strongly coupled. While these two coordinates do couple weakly to all the remaining degrees of freedom, we expect these effects to be relatively small, and that it is the $r_{\mathrm{O}_{\mathrm{d}} \mathrm{H}_{\mathrm{s}}}, r_{\mathrm{O}_{\mathrm{d}} \mathrm{O}_{\mathrm{a}}}$ coupling that will have the most important ramification in the dynamics. Our geometries and energies for the ground state of the water dimer cation compare favorably with a recent study by Cheng et. al. [31], which characterized a variety of stationary points on this surface. A previous theoretical analysis of the geometric and energetic changes associated with the water dimer cation surface reported similar features. In that study, as in ours, the same three major geometric changes were noted as was the strong coupling between the $r_{\mathrm{O}_{\mathrm{d}} \mathrm{H}_{\mathrm{s}}}$ and $r_{\mathrm{O}_{\mathrm{d}} \mathrm{a}}$ motions [32].

The first excited state of the water dimer cation results from the ionization at $13.16 \mathrm{eV}$ of an electron from the $\left(a_{1} / b_{1}\right)^{*}$ orbital, which is an orbital that is delocalized over both the hydrogen bond donor and acceptor. The absence of an initially localized hole deprives the system of the strong driving force for proton transfer that was seen in the ground state.

This ionization also induces large structural changes. The equilibrium structure of the first excited state is also the proton transferred structure, $(\mathrm{OH}) \cdot\left(\mathrm{H}_{3} \mathrm{O}^{+}\right)$. In this structure there are four coordinates displaying large displacements, i.e., the distance between the oxygen atoms, $r_{\mathrm{O}_{\mathrm{d}} \mathrm{O}_{\mathrm{a}}}$, which decreases from $2.85 \AA$ to $2.58 \AA$, the distance between the $\mathrm{OH}$ oxygen and the shared proton, which increases from $0.96 \AA$ to $1.57 \AA$, and the angle between the shared proton, the oxygen in the $\mathrm{OH}$ group, the terminal hydrogen in the $\mathrm{OH}$, which increases from $104^{\circ}$ to $180^{\circ}$ degrees, and the $\mathrm{HOH}$ angle in the donor which also nearly reaches $180^{\circ}$ degrees. The remaining coordinates are not significantly affected by the ionization.

Examining the coupling on the first excited-state cation surface using the same methodology as before reveals a similar strong coupling between the $r_{\mathrm{O}_{\mathrm{d}} \mathrm{H}_{\mathrm{s}}}$ and the $r_{\mathrm{O}_{\mathrm{d}} \mathrm{O}_{\mathrm{a}}}$ coordinates. This is illustrated in Figure 3. An important difference between the ground and first excited state surfaces is also apparent from a comparison of Figure 2 and Figure 3. Whereas on the ground cation surface the vertical ionization places the water dimer in a location where the gradient points toward a proton transferred complex, on the first excited state cation surface 
the vertical ionization places the water dimer on a relatively flat shelf. It is important to note that while the global minimum for both the ground and first excited state of the cation correspond to a proton-transferred complex, the local shape of the PES is profoundly different for the two states. For the ground state the curvature of the local surface leads to quick reaction, whereas for the first excited state the local surface is flat. Of the many stationary points identified by Cheng et. al. [31] only two appear within our criteria for geometrical parameters, these being the global minimum and the transition-state connecting the two identical forms of the global minimum. The important geometrical parameters as well as the energies of each are tabulated in Table I and the geometries are shown in Figure 1.

\section{B. Vibrational Energies}

The ground state cation surface was used for VCI calculations in order to compare with recent experimental and theoretical results. The comparison between experimental vibrational energies from argon-messenger vibrational predissociation spectroscopy [32], our VCI calculated frequencies, and harmonic frequencies from $\operatorname{CCSD}(\mathrm{T}) /$ aug-cc-pVDZ [31] are shown in Table II. Our approach yields substantially more accurate vibrational frequencies because it includes anharmonic effects and allows the motion in different modes to be coupled. This is particularly important for the water dimer cation because certain motions are strongly coupled, i.e., the shared proton stretch and the oxygen-oxygen stretch, and can be seen in the large difference between harmonic and VCI frequencies.

We do not expect perfect agreement because of both the presence of perturbing argon atoms in the experiment and inaccuracies in the PES. Nevertheless, good agreement between the theoretical and experimental values is observed. The previous work by Gardenier et. al. also included harmonic analysis of the fundamental frequencies at the unrestricted MP2/aug-cc-pVDZ level of theory as well as anharmonic reduced-dimensional calculation for the $\nu_{s p(\|)}$ mode. This work also observed a large drop in the frequency of this mode when anharmonicity was accounted for and when it was allowed to couple with the oxygen-oxygen stretch. Our frequencies and assignments are consistent with this previous work and with the later work of Cheng et. al.

The one unexplained feature from the argon-tagged messenger spectroscopy of Gardenier et. al. was a triplet which was tentatively assigned to combination bands involving the 
$\nu_{s p(\|)}$ stretch and the torsional rotation of $\mathrm{H}_{\mathrm{f}}$. While we observe that there are several states with character corresponding to a single excitation in $\nu_{s p(\|)}$, these states are clustered near to our reported value of $1820 \mathrm{~cm}^{-1}$ for that excitation. This makes us suspect that this triplet is a perturbation caused by the presence of the argon atoms. Nevertheless, we cannot definitively rule out the possibility of intensity sharing since we do not at present have a dipole moment surface for the cation and the Watson Hamiltonian has inherent problems with large amplitude rotations which this dihedral rotation may involve.

\section{Photoelectron Spectroscopy}

A photoelectron spectrum for the water dimer shown in Figure 4 was computed using the four-dimensional Hamiltonian given in Eq. (4). The resulting stick spectrum was convoluted with a high-resolution gaussian (full-width at half-maximum of $0.05 \mathrm{eV}$ ). The adiabatic and vertical IEs computed from electronic energy differences are also shown. The two bands in the spectrum correspond to ionization to the ground and first excited states of the water dimer cation. The first feature shows a broad vibrational progression, and is chiefly the result of the overlap of the coupled $r_{\mathrm{O}_{\mathrm{d}} \mathrm{H}_{\mathrm{s}}}, r_{\mathrm{O}_{\mathrm{d}} \mathrm{O}_{\mathrm{a}}}$ vibrational states on the cation ground state surface with the vibrational ground state on the neutral surface. For reference, this spectrum also includes lines for the adiabatic and vertical ionization energies of $11.1 \mathrm{eV}$ and $11.7 \mathrm{eV}$ respectively. The reported vertical ionization is the difference in electronic energies of the neutral water dimer and the cation at the geometry of the neutral dimer. While this yields a value of $11.7 \mathrm{eV}$, the actual peak of the photoelectrom spectrum appears approximately $0.1 \mathrm{eV}$ lower. This shift is due to the combination of better overlap between the vibrational states and the zero-point energy in the Franck-Condon active modes. The

experimental spectrum is limited by the resolution of the measurement and the accuracy of numerical derivation and so the structure apparent in the theoretical spectrum cannot be resolved.

In stark contrast to the ground-state ionization, ionization to the first excited state of the cation does not result in a broad vibrational progression, despite the similarities of the two equilibrium structures. This is because of the difference in the local curvature of the PES in the region accessed by the vertical ionization. As noted above, vertical ionization to the first excited state places the dimer on a broad shelf region supporting a set of localized 
vibrational states. Consequently, the photoelectron spectrum is dominated by a single peak near the vertical ionization at $13.1 \mathrm{eV}$. Because of the poor 0-0 Franck-Condon factor, it is impossible to see the adiabatic onset for this state.

Fig. 5 compares the theoretical spectrum with the experimental pseudo-photoelectron spectrum derived from the PIE curve (see section III). In this region, only the lowest electronic state of the cation is accessible. The onsets and the maxima of the two spectra are in excellent agreement. The spectra exhibit larger differences at higher energies, which might be due to the disappearance of the dimer signal from dissociation. The vertical ionization occurs at $11.7 \mathrm{eV}$, and fragmentation into $\mathrm{H}_{3} \mathrm{O}^{+}+\mathrm{OH}$ becomes possible at 11.8 $\mathrm{eV}$, meaning that the photoelectron spectrum derived from the PIE curve will suffer from attenuation for any energies equal to or greater than this.

\section{Wavepacket Dynamics}

Previous work posited a two-step mechanism for the proton transfer whereby the first step involves the two monomers moving closer together and the second step transfers the proton between the oxygens[30]. Quantum dynamics reveal a more complicated picture for the mechanism, however, as shown in Figure 6. Immediately upon ionization, the wave packet is located at a region on the cation PES which simultaneously begins driving the monomermonomer distance to decrease and the proton to transfer. Because the proton moves on a significantly faster timescale than the heavy fragments, it oscillates between the two oxygens as the monomers gradually close the gap. Thus there are several proton transfer events and this is more consistent with a picture where the hydrogen oscillates back and forth between the two oxygens until they have moved close enough to trap it in a vibrationally excited $\mathrm{H}_{4} \mathrm{O}_{2}^{+}$. The expectation values of the two coordinates are shown in Figure 7 and at about 50 femtoseconds, both values have reached the point where the reaction of $\mathrm{H}_{2} \mathrm{O}^{+}$and $\mathrm{H}_{2} \mathrm{O}$ can be considered complete. (The presence of dissipative forces in the bulk may somewhat slow the process, but we expect it to be complete on roughly this timescale.)

The proton transfer dynamics also manifests itself in the photoelectron spectrum. The vibrational progression with several distinct peaks indicates that there is a set of vibrational states which overlap with the ground vibrational state of the neutral water dimer. It is the 
evolution of these cation vibrational states which drives the subsequent dynamics on that surface.

The dynamics on the ground-state cation surface are shown in Figure 8 and can be contrasted with the wave packet evolution on the first excited-state surface. Looking at the photoelectron spectrum for this state (Figure 4), we see that it is dominated by a single peak. This indicates that the neutral water vibrational ground state is well represented by relatively few localized vibrational states of the electronically excited cation. The subsequent propagation should show only slow spreading of the wavepacket and little movement because of the flatness of the local surface. These suppositions are realized during the propagation as shown in Figure 8.

\section{E. Electronic Spectral Evolution}

As the wavepacket moves towards the products, the electronic spectrum of the cation also evolves as shown in Figure 9. The spectrum shown in Figure 9 is best viewed as a two laser experiment, where the first laser instantaneously ionizes the neutral cation, and the second laser interrogates the transition of the cation at a specific excitation energy. The time delay between the two pulses corresponds to the evolution time of the wavepacket and is thus given by the $x$-axis in the top panel of Figure 9 .

An interesting feature of Figure 9 is the increase in the intensity of the transition between the ground and the third excited state of $\mathrm{H}_{4} \mathrm{O}_{2}^{+}$. In the DMO-LCFMO terms, the ground state corresponds to ionization from the $b_{1} / 0$ orbital while the third excited state corresponds to ionization from the $\left(b_{2} / a_{1}\right)^{*}$ orbital. The transition between the ground state and third excited state is therefore derived by exciting from the doubly occupied $\left(b_{2} / a_{1}\right)^{*}$ orbital to the $b_{1} / 0$ singly occupied molecular orbital (SOMO). As the cation evolves from the vertical geometry towards the proton transferred geometry, the shared proton moves closer to the hydrogen-bond acceptor causing mixing between the $b_{1}$ and $a_{1}$ fragment molecular orbitals. This causes the $\left(b_{2} / a_{1}\right)^{*}$ orbital, which was initially anti-bonding in character with respect to the fragments, to become bonding. Coupled with the rotation of the hydrogen-bond donor group, this allows the transition to this excited state to acquire significantly more intensity than was originally present.

A similar spectrum computed for the wave packet evolving on the first excited state 
is shown in Figure 10. This spectrum is dominated by the transition between the first and second excited states. This corresponds to exciting an electron from the $a_{1} / b_{1}$ doubly occupied molecular orbital to the $\left(a_{1} / b_{1}\right)^{*}$ singly occupied molecular orbital. Because the evolution on this surface shows little movement of the wavepacket, the spectrum shows little change as well. Towards the end of the propagation as the shared hydrogen moves slightly farther away from the $\mathrm{OH}$ end of the complex, the second and fourth excited states are pushed slightly higher in energy, but there is little change in the transition strengths.

\section{CONCLUSIONS}

This study reveals that the dynamics of the proton transfer process can be highly dependent, both qualitatively and quantitatively, on the nature of the ionized state. In the water dimer, the proton transfer is driven by the localization of the hole on one individual monomer. In the ground state of the cation, the hole is initially strongly localized on the hydrogen-bond donating monomer, and subsequent propagation of the vertically ionized wavepacket reveals several proton transfer events as the hole moves in opposition to the proton. This is in contrast to the case where the ionization places the cation on the first excited state. In this case the hole is delocalized over both the hydrogen-bond donor and acceptor and the dynamics reveal little movement of the wavepacket. These differences are also responsible for different shapes of the two lowest bands of the photoelectron spectrum.

This study illustrates the markedly different behavior between different states of the cation, provides an estimation of the reaction time in terms of wavepacket dynamics, and offers a potential aid to the detection of this transfer by calculating the evolution of the spectral lines during the event. It also raises some questions about the dynamics of the proton transfer event in larger water clusters or in bulk water, which could be addressed in a future study.

By combining state-of-the-art electronic structure calculations with wavepacket dynamics we are able to accurately capture and help interpret all the relevant points of the experimental spectra. Our calculations accurately reproduce the spectra as well as yield frequencies that are in good agreement with the observed values. Within this context it is important to note the importance of including anharmonic effects in the calculation of vibrational frequencies, which can significantly lower the frequency from the calculated harmonic value. For the 
water dimer cation, the shared proton stretch and the oxygen-oxygen stretch are strongly coupled yielding large anharmonic effects. We also emphasize the importance of modeling the entire photoelectron spectrum, as our calculations show that the Franck-Condon factors can entirely hide the adiabatic onset of ionization as well as shift the peak to the left of the vertical value. This means that extra care must be taken in interpreting these features in experimental spectra since the peak may be shifted slightly from the vertical ionization energy and the adiabatic onset may not be visible.

\section{Acknowledgment}

This work is conducted under auspices of the iOpenShell Center for Computational Studies of Electronic Structure and Spectroscopy of Open-Shell and Electronically Excited Species supported by the National Science Foundation through the CRIF:CRF CHE0625419+0624602+0625237 grant. AIK and JMB also acknowledge support of the Department of Energy (DE-FG02-05ER15685 and DE-FG02-97ER14782, respectively). MA acknowledges support by the Director, Office of Energy Research, Office of Basic Energy Sciences, Chemical Sciences Division of the U.S. Department of Energy under contract No. DE-AC02-05CH11231.

[1] Bruce C Garrett et al., Chem Rev 105, 355 (2005).

[2] J. A. LaVerne and S. M. Pimblott, J. Phys. Chem. A 101, 5828 (1997).

[3] J. A. LaVerne and S. M. Pimblott, J. Phys. Chem. 96, 8905 (1992).

[4] F. C. Fehsenfeld, M. Mosesman, and E. E. Ferguson, J. Chem. Phys. 55, 2115 (1971).

[5] L. Angel and A. J. Stace, J. Phys. Chem. A 103, 2999 (1999).

[6] L.P. Candeias and S. Steenken, J. Am. Chem. Soc. 111, 1094 (1989).

[7] S. Steenken, Chem. Rev. 89, 503 (1989).

[8] A.-O. Colson, B. Besler, and M.D. Sevilla, J. Phys. Chem. 96, 9787 (1992).

[9] M. Hutter and T. Clark, J. Am. Chem. Soc. 118 (1996).

[10] A.K. Ghosh and G.B. Schuster, J. Am. Chem. Soc. 128, 4172 (2006).

[11] A. Kumar and M.D. Sevilla, J. Phys. Chem. B (2009), asap. 
[12] J. Bertran, A. Oliva, L. Rodríguez-Santiago, and M. Sodupe, J. Am. Chem. Soc. 120, 8159 (1998).

[13] K.B. Bravaya, O. Kostko, M. Ahmed, and A.I. Krylov, Phys. Chem. Chem. Phys. (2010), in press, DOI:10.1039/b919930f.

[14] A.A. Zadorozhnaya and A.I. Krylov, J. Chem. Theory Comput. (2009), submitted.

[15] A.L. Sobolewski, W. Domcke, and C. Hättig, Proc. Nat. Acad. Sci. 102, 17903 (2005).

[16] S. Perun, A.L. Sobolewski, and W. Domcke, J. Phys. Chem. A 110, 9031 (2006).

[17] Y. Gauduel, S. Pommeret, A. Migus, and A. Antonetti, Chem. Phys. 149, 1 (1990).

[18] K. Liu, J. D. Cruzan, and R. J. Saykally, Science 271, 929 (1996).

[19] J. K. Gregory, D. C. Clary, K. Liu, M. G. Brown, and R. J. Saykally, Science 275, 814 (1997).

[20] R. N. Barnett and U. Landman, Phys. Rev. B 48, 2081 (1993).

[21] X. Huang, B.J. Braams, and J. M. Bowman, J. Phys. Chem. A 110, 445 (2006).

[22] A. Shank, Y. Wang, A. Kaledin, B. J. Braams, and J. M. Bowman, J. Chem. Phys. 130, $144314(2009)$.

[23] D. Moncrieff, I.H. Hillier, and V.R. Saunders, Chem. Phys. Lett. 89, 447 (1982).

[24] L.A. Curtiss, Chem. Phys. Lett. 96, 442 (1983).

[25] P.M.W. Gill and L. Radom, J. Am. Chem. Soc. 110, 4931 (1988).

[26] M. Sodupe, A. Oliva, and J. Bertran, J. Am. Chem. Soc. 116, 8249 (1994).

[27] A. Furuhama, M. Dupuis, and K. Hirao, J. Chem. Phys. 124, 164310 (2006).

[28] R.N. Barnett and U. Landman, J. Phys. Chem. 99, 17305 (1995).

[29] P.A. Pieniazek, J. VandeVondele, P. Jungwirth, A.I. Krylov, and S.E. Bradforth, J. Phys. Chem. A 112, 6159 (2008).

[30] P.A. Pieniazek, E.J. Sundstrom, S.E. Bradforth, and A.I. Krylov, J. Phys. Chem. A 113, $4423(2009)$.

[31] Q. Cheng, F.A. Evangelista, A.G. Simmonett, Y. Yamaguchi, and H.F. Schaefer, J. Phys. Chem. A (2009), in press, ASAP article.

[32] G. H. Gardenier, M. A. Johnson, and A. B. McCoy, J. Phys. Chem. A 113, 4772 (2009).

[33] Y. Achiba S. Tomoda and K. Kimura, Chem. Phys. Lett. 87, 197 (1982).

[34] C.Y. Ng, D.J. Trevor, P.W. Tiedemann, S.T. Ceyer, P.L. Kronebusch, B.H. Mahan, and Y.T. Lee, J. Chem. Phys. 67, 4235 (1977).

[35] S. P. de Visser, L.J. de Koning, and N.M.M. Nibbering, J. Phys. Chem. 99, 15444 (1995). 
[36] S. Barth, M. Ončák, V. Ulrich, M. Mucke, T. Lischke, and P. Slavíček, J. Phys. Chem. A (2009), in press, ASAP article.

[37] S. Pal, M. Rittby, R.J. Bartlett, D. Sinha, and D. Mukherjee, Chem. Phys. Lett. 137, 273 (1987).

[38] J.F. Stanton and J. Gauss, J. Chem. Phys. 101, 8938 (1994).

[39] M. Kamiya and S. Hirata, J. Chem. Phys. 125, 074111 (2006).

[40] P.A. Pieniazek, S.A. Arnstein, S.E. Bradforth, A.I. Krylov, and C.D. Sherrill, J. Chem. Phys. 127, $164110(2007)$.

[41] P.A. Pieniazek, S.E. Bradforth, and A.I. Krylov, J. Chem. Phys. 129, 074104 (2008).

[42] A.A. Golubeva and A.I. Krylov, Phys. Chem. Chem. Phys. 11, 1303 (2009).

[43] O. Kostko, K.B. Bravaya, A.I. Krylov, and M. Ahmed, Phys. Chem. Chem. Phys. (2009), submitted.

[44] A.I. Krylov, Annu. Rev. Phys. Chem. 59, 433 (2008).

[45] P.A. Pieniazek, A.I. Krylov, and S.E. Bradforth, J. Chem. Phys. 127, 044317 (2007).

[46] C.M. Oana and A.I. Krylov, J. Chem. Phys. 127, 234106 (2007).

[47] B. J. Braams and J. M. Bowman, Int. Rev. Phys. Chem. 28, 577 (2009).

[48] T.H. Dunning, J. Chem. Phys. 90, 1007 (1989).

[49] Y. Shao, L.F. Molnar, Y. Jung, J. Kussmann, C. Ochsenfeld, S. Brown, A.T.B. Gilbert, L.V. Slipchenko, S.V. Levchenko, D.P. O’Neil, R.A. Distasio Jr, R.C. Lochan, T. Wang, G.J.O. Beran, N.A. Besley, J.M. Herbert, C.Y. Lin, T. Van Voorhis, S.H. Chien, A. Sodt, R.P. Steele, V.A. Rassolov, P. Maslen, P.P. Korambath, R.D. Adamson, B. Austin, J. Baker, E.F.C. Bird, H. Daschel, R.J. Doerksen, A. Drew, B.D. Dunietz, A.D. Dutoi, T.R. Furlani, S.R. Gwaltney, A. Heyden, S. Hirata, C.-P. Hsu, G.S. Kedziora, R.Z. Khalliulin, P. Klunziger, A.M. Lee, W.Z. Liang, I. Lotan, N. Nair, B. Peters, E.I. Proynov, P.A. Pieniazek, Y.M. Rhee, J. Ritchie, E. Rosta, C.D. Sherrill, A.C. Simmonett, J.E. Subotnik, H.L. Woodcock III, W. Zhang, A.T. Bell, A.K. Chakraborty, D.M. Chipman, F.J. Keil, A. Warshel, W.J. Herhe, H.F. Schaefer III, J. Kong, A.I. Krylov, P.M.W. Gill, M. Head-Gordon, Phys. Chem. Chem. Phys. 8, 3172 (2006).

[50] E. Kamarchik, B. Braams, A.I. Krylov, and J.M. Bowman, ezPES, http://iopenshell.usc.edu/downloads/.

[51] J. M. Bowman, T. Carrington, and H.-D. Meyer, Mol. Phys. 106, 2145 (2008). 
[52] J. M. Bowman, J. Chem. Phys. 68, 608 (1978).

[53] A. S. Dickinson and P. R. Certain, J. Chem. Phys. 49, 4209 (1968).

[54] J. C. Light and T. Carrington, in Advances in Chemical Physics, Vol 114, volume 114 of Advances in Chemical Physics, pages 263-310. 2000.

[55] J. P. Boyd, Chebyshev and Fourier Spectral Methods: Second Revised Edition. Dover Publications, 2001.

[56] J. K. G. Watson, Mol. Phys. 15, 479 (1968).

[57] J. C. Decius, J. Chem. Phys. 16, 1025 (1948).

[58] See EPAPS document No. for the molecular structures and energies. This document can be reached through a direct link in the online article. HTML reference section or via the EPAPS homepage (http://www.aip.org/pubservs/epaps.html).

[59] J. Berkowitz, J. Chem. Phys. 69, 3044 (1978). 
TABLE I: Geometrical parameters and energies of the important geometries for $\mathrm{H}_{4} \mathrm{O}_{2}^{+}$in the ground and the first excited state. Lengths are given in $\AA$ and energies are given in $\mathrm{kcal} / \mathrm{mol}$.

\begin{tabular}{cccccccc}
\hline \hline Structure & $r_{\mathrm{OO}}$ & $r_{\mathrm{OHs}}$ & $\theta_{\mathrm{HsOH}}$ & $\tau_{\mathrm{HOHO}}$ & $\mathrm{H}_{4} \mathrm{O}_{2}$ & $\mathrm{H}_{4} \mathrm{O}_{2}^{+}$ & $\mathrm{H}_{4} \mathrm{O}_{2}^{+*}$ \\
\hline vertical & 2.923 & 0.965 & $104.6^{\circ}$ & $180.0^{\circ}$ & 0.0 & 270.3 & 301.2 \\
$\mathrm{H}_{4} \mathrm{O}_{2}^{+}$minimum & 2.510 & 1.465 & $119.8^{\circ}$ & $147.2^{\circ}$ & & 248.8 & \\
$\mathrm{H}_{4} \mathrm{O}_{2}^{+}$ts & 2.538 & 1.488 & $120.3^{\circ}$ & $180.0^{\circ}$ & 248.9 & \\
$\mathrm{H}_{4} \mathrm{O}_{2}^{+*}$ minimum & 2.577 & 1.569 & $180.0^{\circ}$ & $180.0^{\circ}$ & & & 252.1 \\
\hline \hline
\end{tabular}


TABLE II: Comparison of experimental frequencies from vibrational predissociation spectroscopy[32], theoretical VCI frequencies, and the harmonic results from Cheng et. al.[31]. Frequencies are given in $\mathrm{cm}^{-1}$.

\begin{tabular}{cccc}
\hline \hline Mode & VCI & Experiment & Harmonic CCSD $(\mathrm{T}) /$ aug-cc-pVQZ \\
\hline$\nu_{\text {bend }}$ & 1536 & $1543^{a}$ & 1658 \\
$\nu_{s p(\perp)}$ & 1648 & & 1719 \\
$\nu_{s p(\|)}$ & 1820 & $1810^{b}$ & 2373 \\
$\nu_{\mathrm{OH}}^{\mathrm{sym}}$ & 3508 & $3392^{a}$ & 3702 \\
$\nu \cdot \mathrm{OH}$ & 3530 & $3511^{a}$ & 3666 \\
$\nu_{\mathrm{OH}}$ & 3604 & $3591^{a}$ & 3780 \\
$2 \nu_{s p(\|)}$ & 3280 & $3270^{a}$ & \\
\hline \hline
\end{tabular}

${ }^{a}$ Frequencies are for $\mathrm{H}_{4} \mathrm{O}_{2}^{+} \cdot \mathrm{Ar}$.

${ }^{b}$ Extrapolated frequency for $\mathrm{H}_{4} \mathrm{O}_{2}^{+}$. 


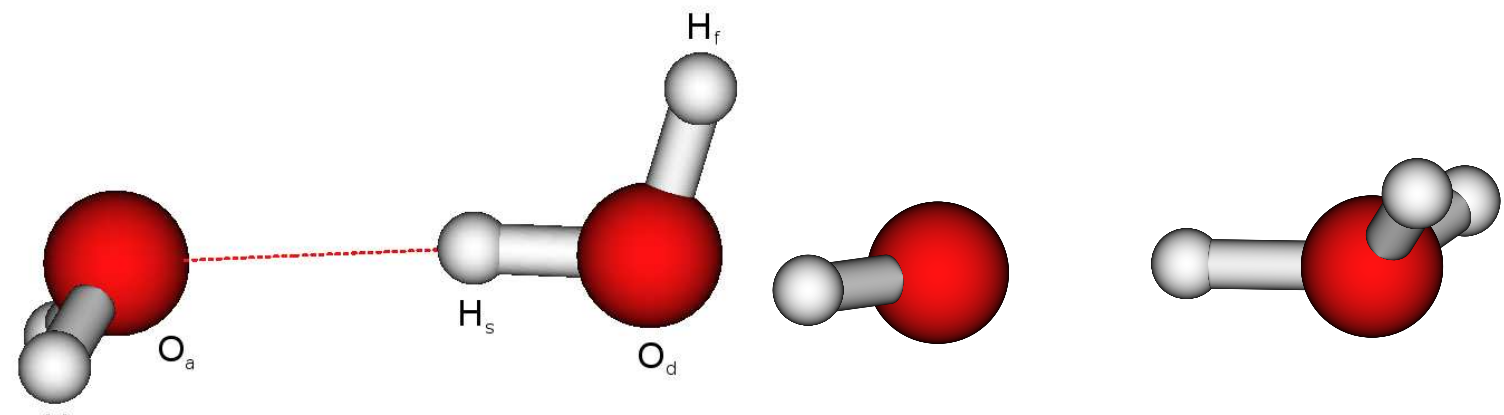

$\mathrm{H}_{\mathrm{a}}$
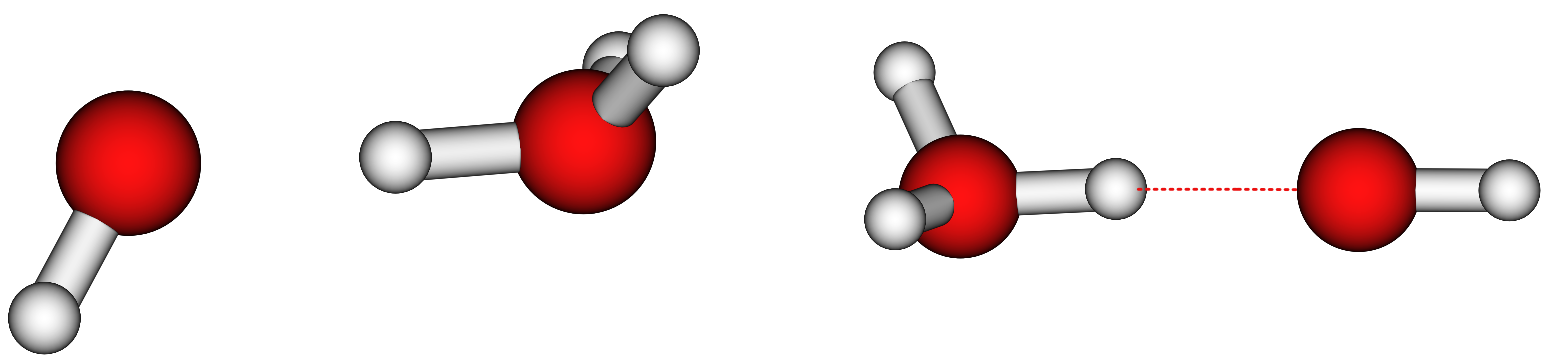

FIG. 1: The four important geometries on the potential energy surface. Starting clockwise from the upper-left hand corner they are the vertical geometry [global minimum of $\left(\mathrm{H}_{2} \mathrm{O}\right)_{2}$ ], the global minimum of $\left(\mathrm{H}_{2} \mathrm{O}\right)_{2}^{+}$, the transition state of $\left(\mathrm{H}_{2} \mathrm{O}\right)_{2}^{+}$, and the global minimum of $\left(\mathrm{H}_{2} \mathrm{O}\right)_{2}^{+*}$. 

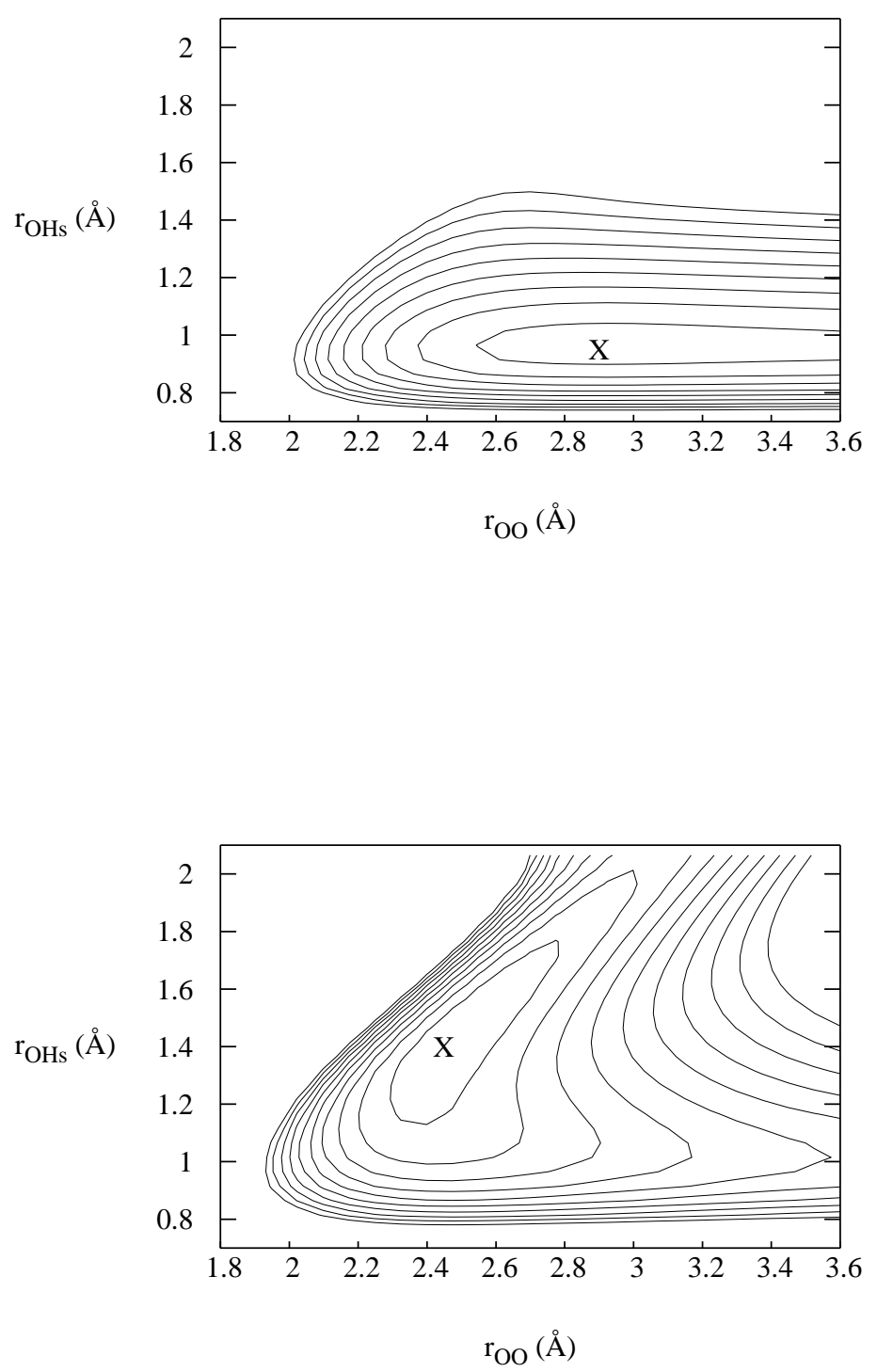

FIG. 2: The two-dimensional slice of the PES of the neutral $\left(\mathrm{H}_{2} \mathrm{O}\right)_{2}$, top, and ground state cation $\left(\mathrm{H}_{2} \mathrm{O}\right)_{2}^{+}$, bottom, as a function of the coordinates $r_{\mathrm{OH}, \mathrm{s}}$ and $r_{\mathrm{OO}}$. While these two coordinates are mostly uncoupled in the neutral dimer, they become strongly coupled in the cation. The minimum on each surface is indicated by an ' $\mathrm{X}$ ' on the figure. 


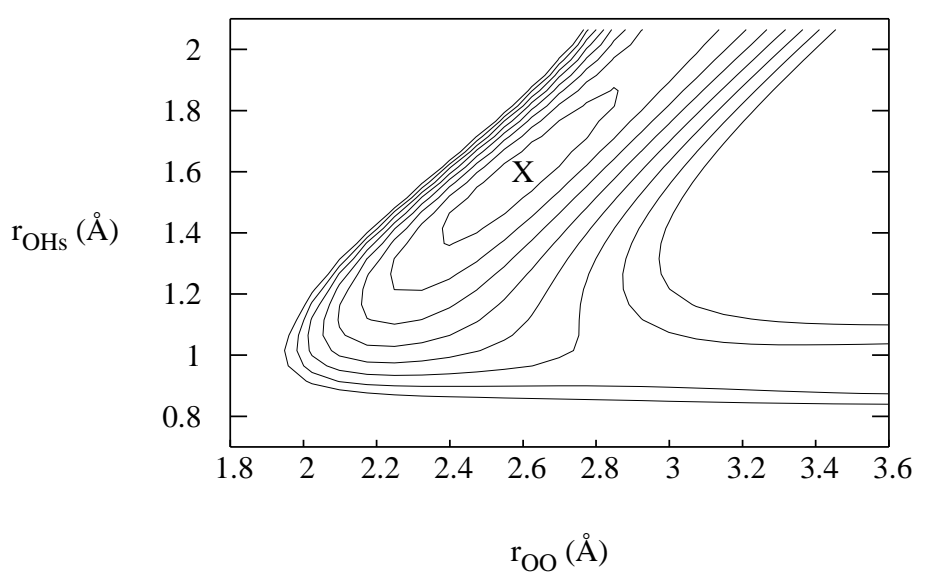

FIG. 3: The PES of the first excited state of the cation as a function of the coordinates $r_{\mathrm{OH}, \mathrm{s}}$ and $r_{\mathrm{OO}}$. While the minimum, indicated by an 'X', still corresponds to a proton transferred geometry, there is a shelf in the Franck-Condon region, resulting in slow proton transfer in this state. 


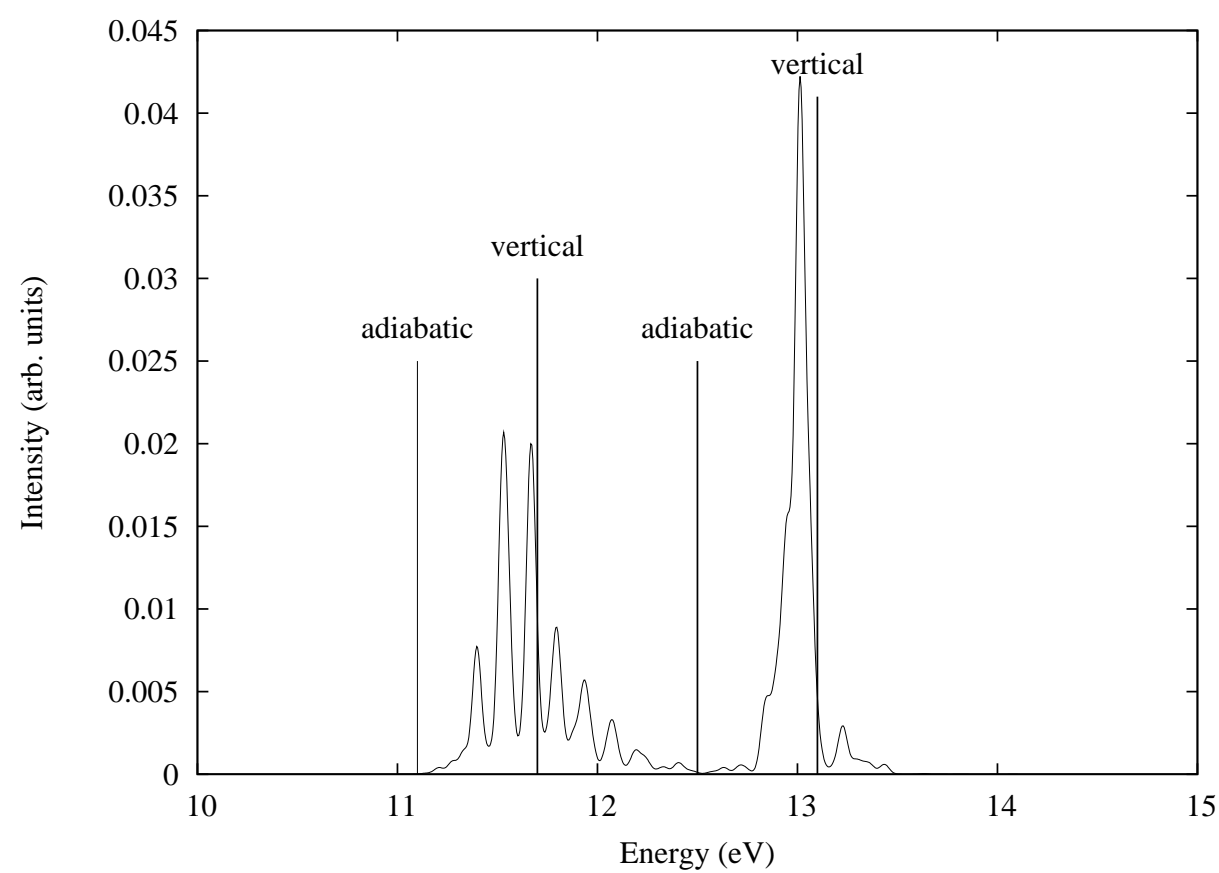

FIG. 4: The calculated photoelectron spectrum of the water dimer corresponding to the ground state of the cation. There is a broad vibrational progression corresponding primarily to excited vibrational states of the coupled $\mathrm{O}-\mathrm{O}$ and $\mathrm{O}-\mathrm{H}$ stretches. The first feature has an onset of $11.1 \mathrm{eV}$ and peaks at $11.7 \mathrm{eV}$, which are the adiabatic and vertical IEs, respectively. The second feature corresponds to the first excited state of the cation and has a single peak near the vertical ionization at $13.1 \mathrm{eV}$ 


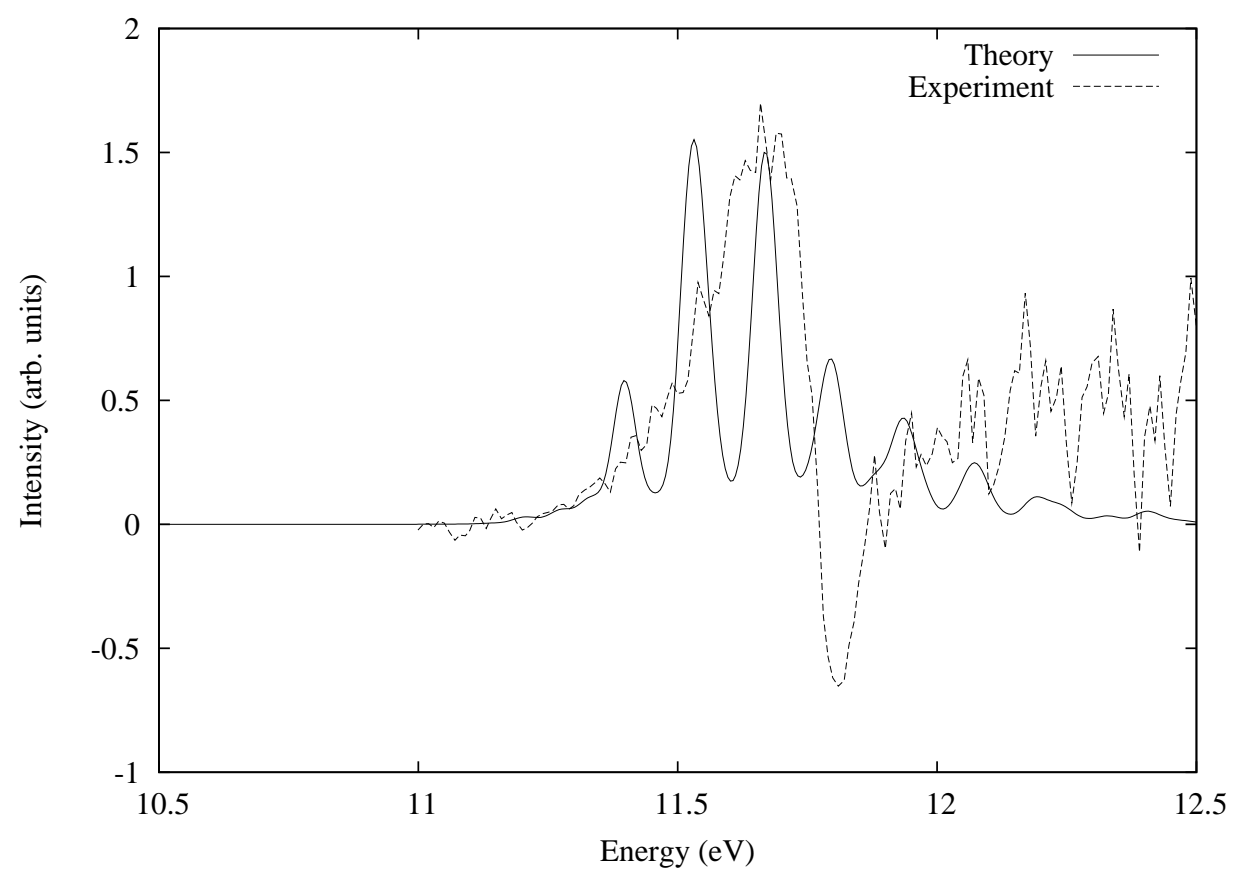

FIG. 5: Comparison of theoretical and experimental photoelectron spectra of the water dimer. The experimental spectrum is derived by taking the numeric derivative of averaged PIE curves, as fragment channels become energetically available the PIE curve can decrease resulting in regions of negative slope. 

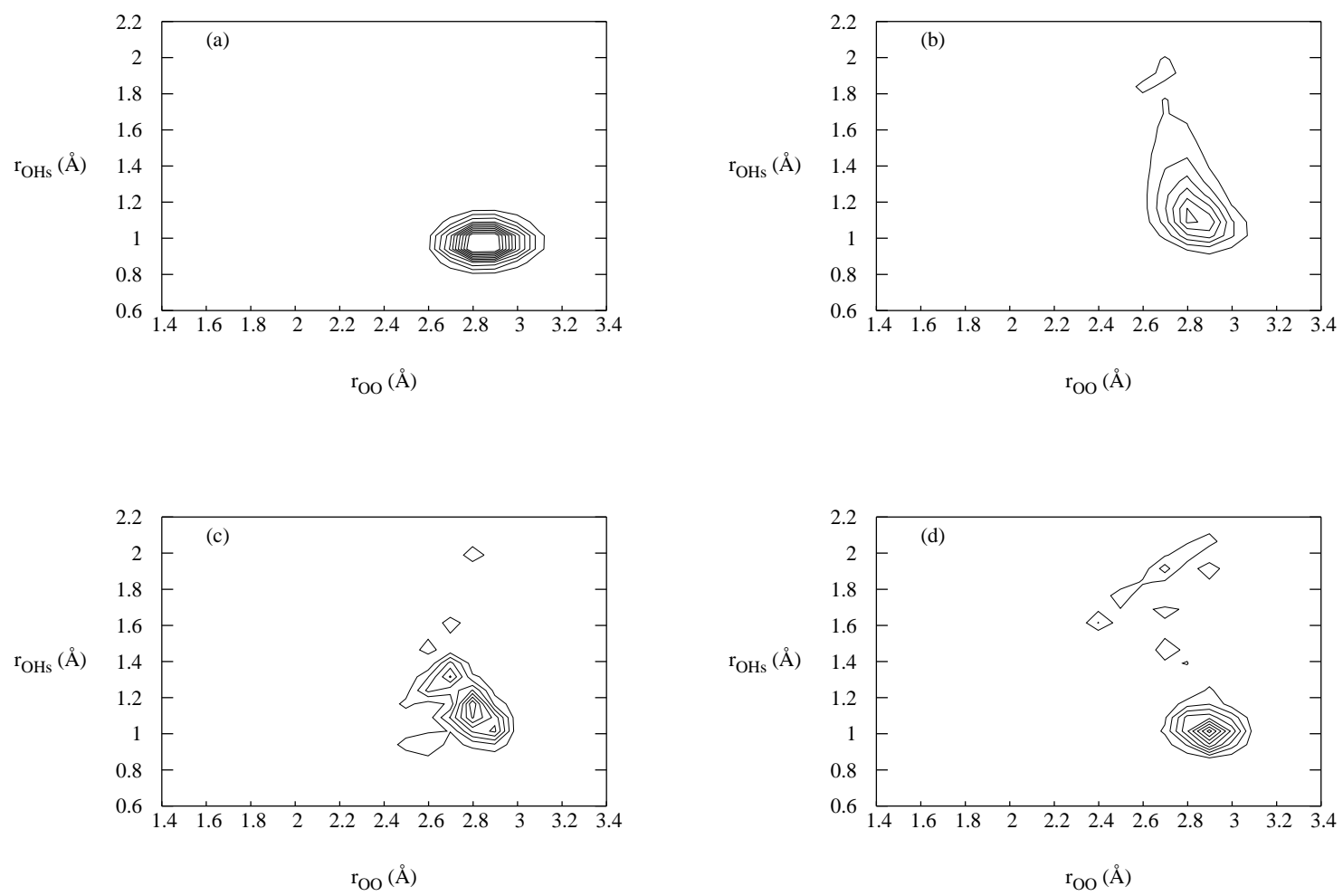

FIG. 6: Projection of the ground-state cation wave packet on the two-dimensional subspace of the $r_{\mathrm{OH}, \mathrm{s}}$ and $r_{\mathrm{OO}}$ coordinates. Starting from the initial vertically ionized position, (a), the wavepacket begins spreading along the $r_{\mathrm{OH}, \mathrm{s}}$ coordinate in (b). Because the $r_{\mathrm{OO}}$ distance is still relatively unchanged, the wavepacket encounters a small barrier and the resulting transmission and reflection can be seen in (c) and (d). 


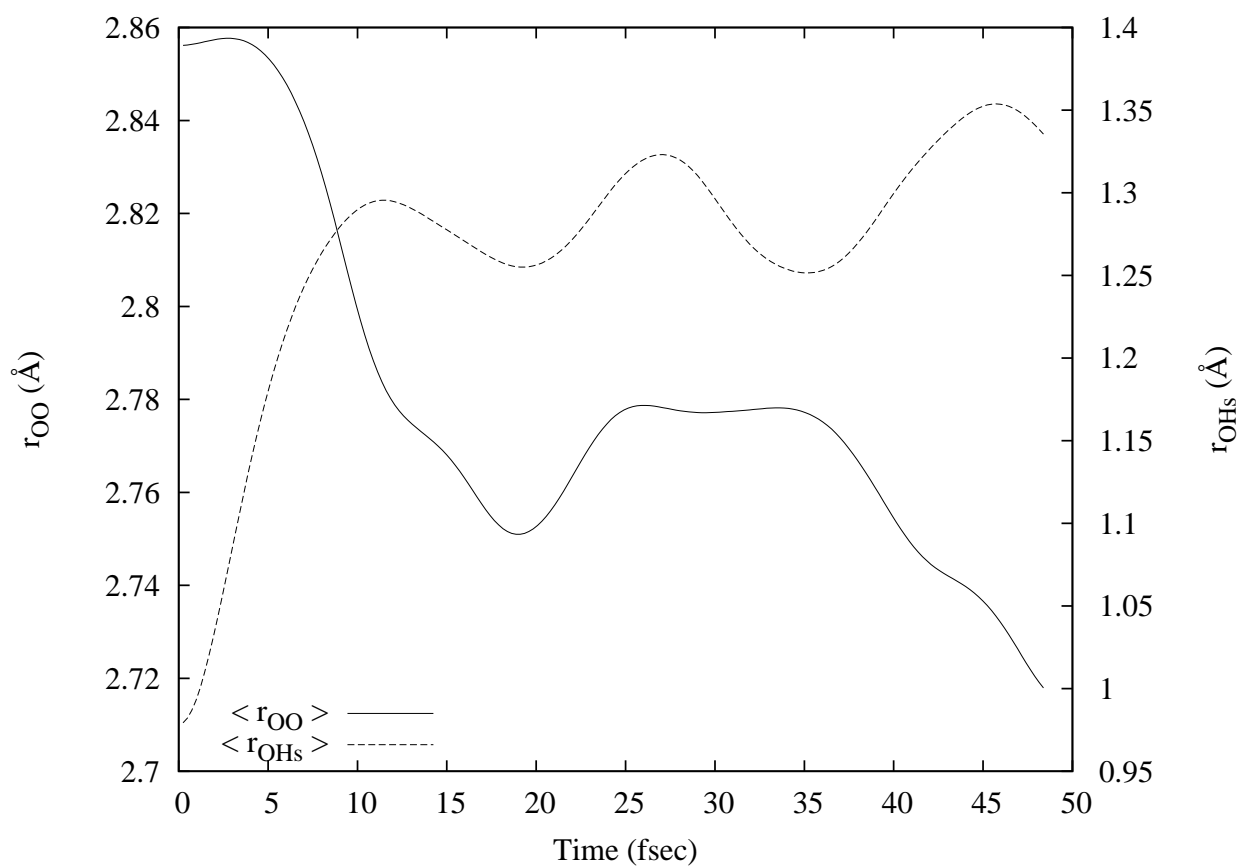

FIG. 7: The expectation values of the coordinates $r_{\mathrm{OH}, \mathrm{s}}$ and $r_{\mathrm{OO}}$ as a function of wave packet propagation time. The hydrogen moves on a significantly faster timescale than the monomer and is able to complete approximately three oscillations in the same time required for the monomers to move closer. At about 50 femtoseconds, the wave packet has arrived at the product state of the $(\mathrm{OH}) \cdot\left(\mathrm{H}_{3} \mathrm{O}\right)^{+}$complex. 

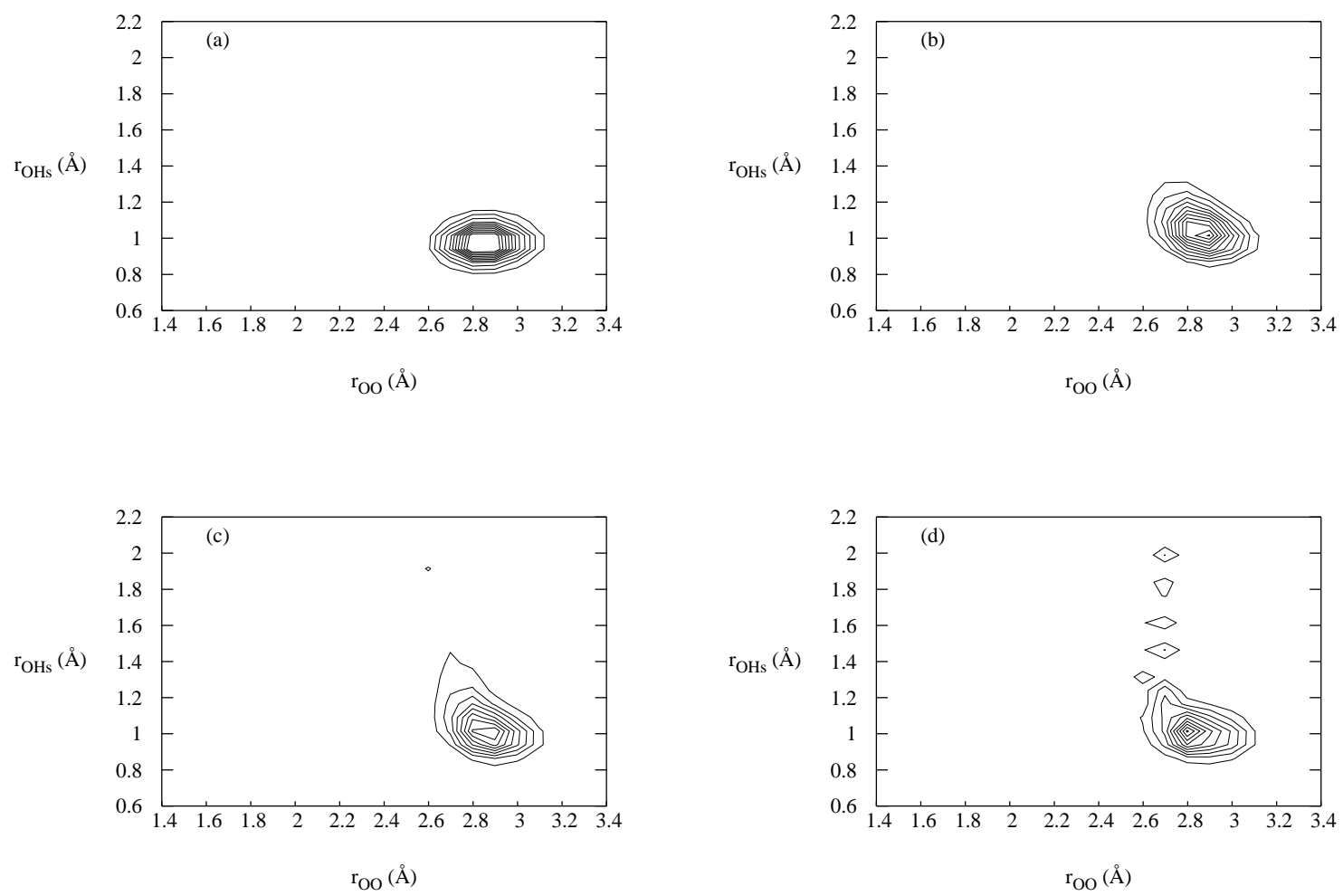

FIG. 8: Projection of the excited-state cation wave packet on the two-dimensional subspace of the $r_{\mathrm{OH}, \mathrm{s}}$ and $r_{\mathrm{OO}}$ coordinates. The propagation of the wave packet is shown at several time steps after the initial vertical ionization, (a). Because of the flatness of the potential energy surface in the region of the vertical transition, the wavepacket remains relatively localized during the subsequent propagation, (b), (c), and (d). 


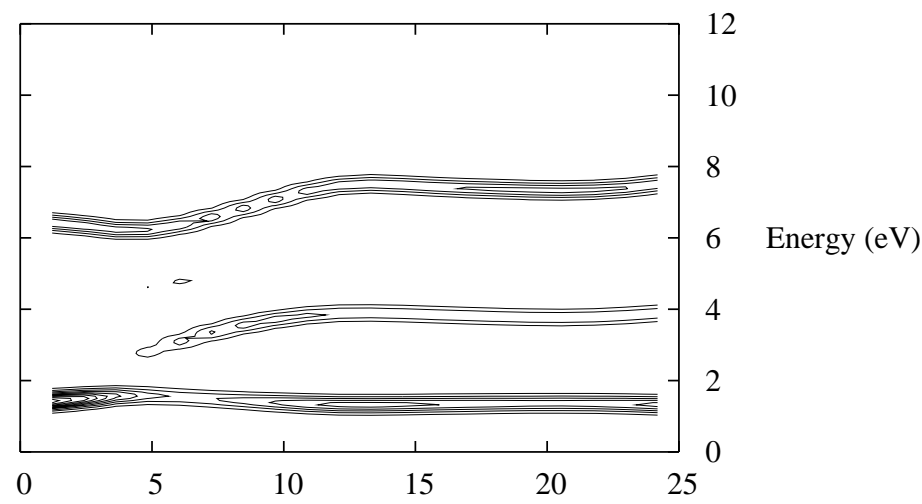

Time (fs)

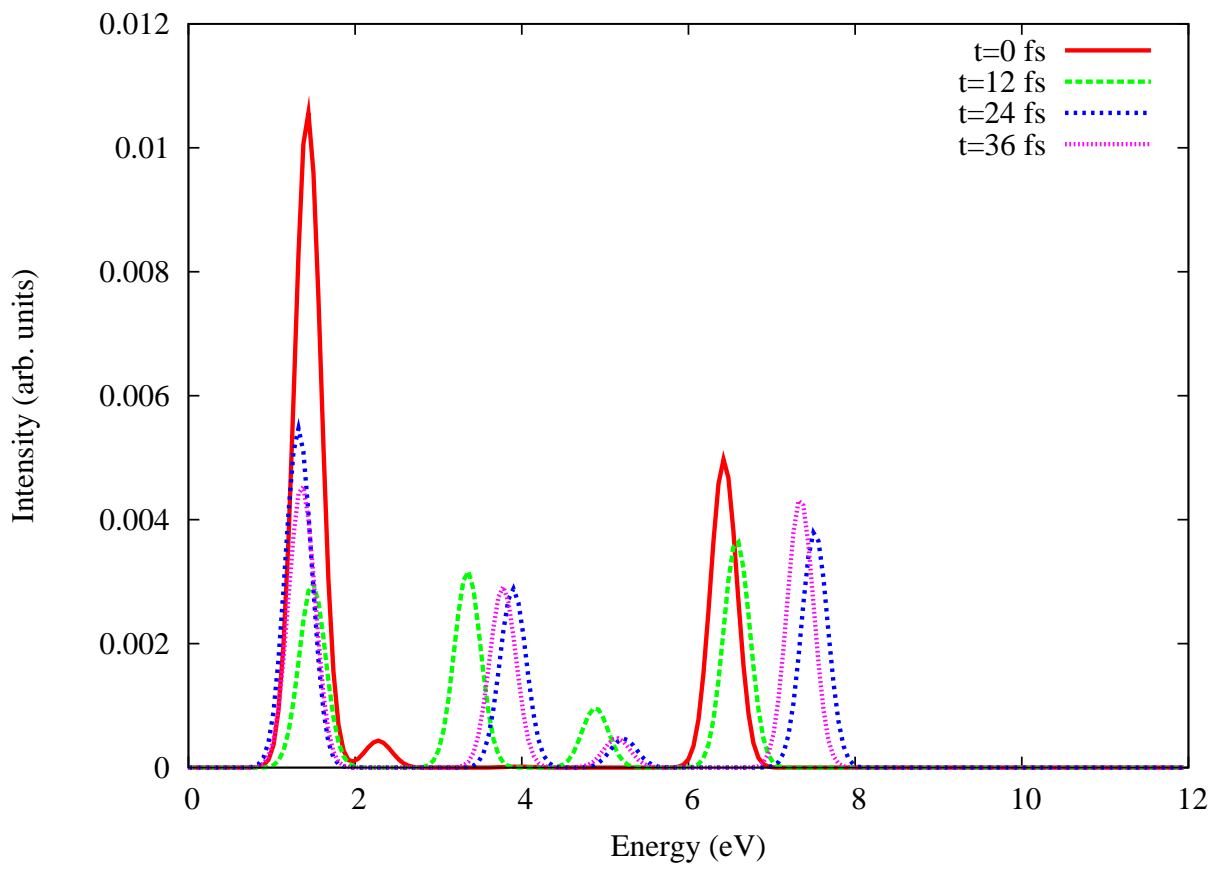

FIG. 9: The spectral evolution of the excited states of $\mathrm{H}_{4} \mathrm{O}_{2}^{+}$when the dynamics takes place on the ground state surface of the cation. The contours correspond to the intensities of individual electronic transitions. Excitation energies and transition dipole moments are evaluated at single point geometries along the path of the wave packet centroid. 


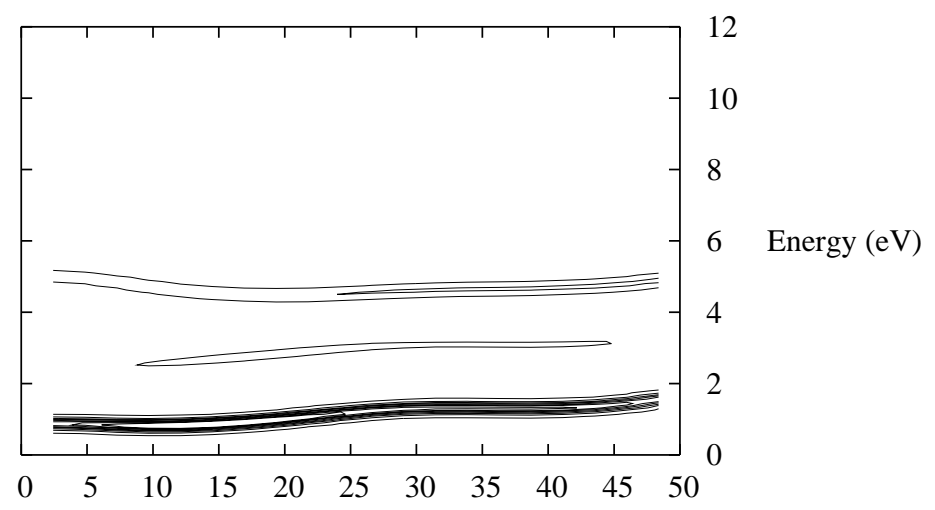

Time (fs)

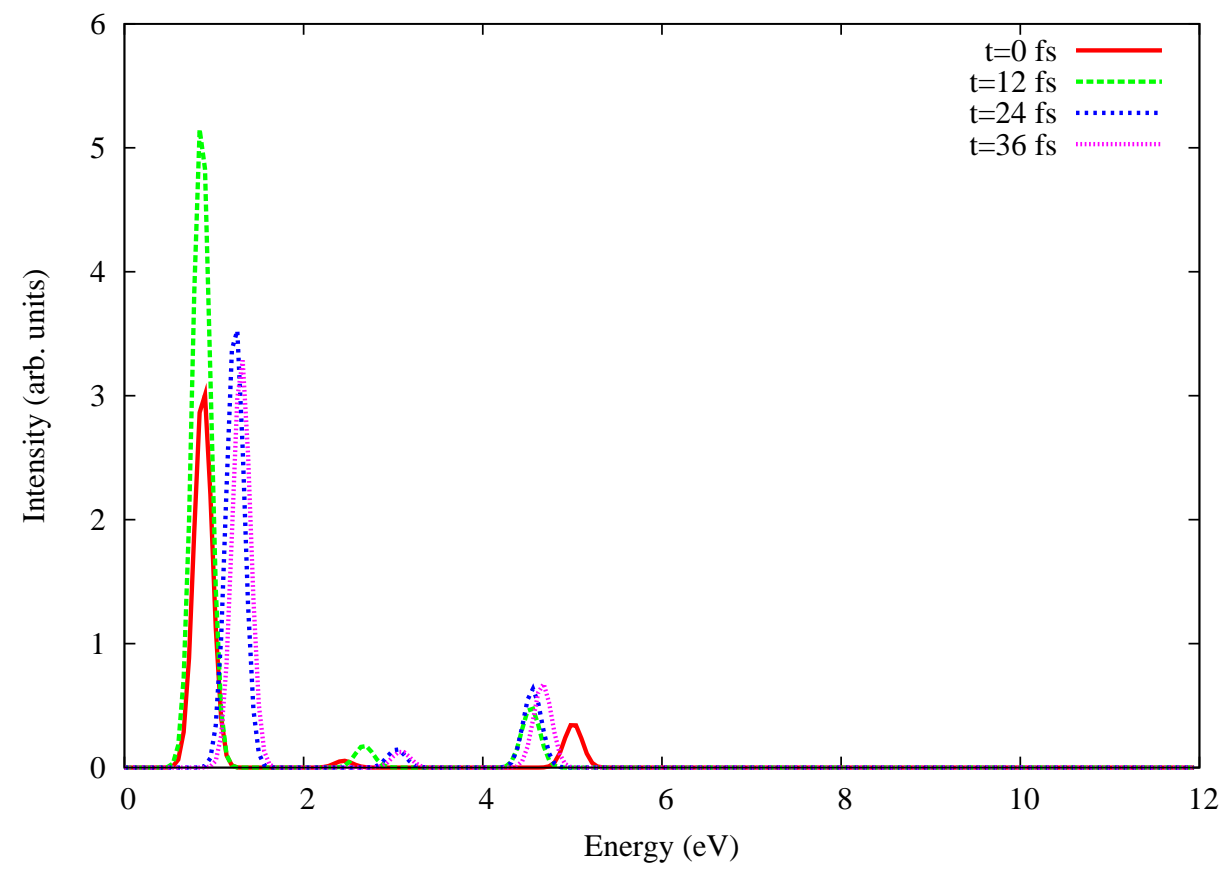

FIG. 10: The spectral evolution of the excited states of $\mathrm{H}_{4} \mathrm{O}_{2}^{+}$when the dynamics takes place on the excited state surface of the cation. The contours correspond to the intensities of individual electronic transitions. Excitation energies and transition dipole moments are evaluated at single point geometries along the path of the wave packet centroid. 